\title{
(2) OPEN ACCESS \\ Molecular characterisation and antibiotic susceptibility of meningococcal isolates from healthy men who have sex with men
}

\author{
Arianna Neri $\odot,{ }^{1}$ Annapina Palmieri, ${ }^{1}$ Grazia Prignano, ${ }^{2}$ Massimo Giuliani, ${ }^{3}$ \\ Alessandra Latini, ${ }^{3}$ Cecilia Fazio, ${ }^{1}$ Paola Vacca, ${ }^{1}$ Luigina Ambrosio, ${ }^{1}$ \\ Andrea Ciammaruconi, ${ }^{4}$ Silvia Fillo, ${ }^{4}$ Anna Anselmo, ${ }^{4}$ Antonella Fortunato, ${ }^{4}$ \\ Romano Lista, ${ }^{4}$ Paola Stefanelli ${ }^{1}$
}

\begin{abstract}
'Department of Infectious Diseases, Istituto Superiore di Sanità, Roma, Italy ${ }^{2}$ Clinical Pathology and Microbiology Unit, IRCC San Gallicano Institute, Rome, Italy ${ }^{3}$ STI/HIV Unit, San Gallicano Dermatological Institute IRCCS, Istituto Dermatologico San Gallicano, Roma, Italy ${ }^{4}$ Scientific Department, Army Medical Center, Centro Studi e Ricerche di Sanita' e Veterinaria dell' Esercito, Roma, Italy
\end{abstract}

Correspondence to

Dr Arianna Neri, Department Infectious Diseases, Istituto Superiore di Sanità, Roma, Italy; arianna.neri@iss.it

$\mathrm{AN}$ and $\mathrm{AP}$ contributed equally.

Received 30 June 2021 Accepted 31 October 202

Check for updates

(c) Author(s) (or their employer(s)) 2021. Re-use permitted under CC BY-NC. No commercial re-use. See rights and permissions. Published by BMJ.

To cite: Neri A, Palmieri A, Prignano $\mathrm{G}$, et al. Sex Transm Infect Epub ahead of print: [please include Day Month Year]. doi:10.1136/ sextrans-2021-055173

\section{ABSTRACT \\ Objectives To evaluate and characterise}

meningococcal carriage among healthy men who have sex with men (MSM) within a screening programme for Neisseria gonorrhoeae infection at the San Gallicano Dermatological Institute, Italy.

Methods A total of 441 MSM attending the STI/ HIV Centre of the San Gallicano Institute, Rome, Italy, in 2016 were routinely screened for $N$. gonorrhoeae infection by pharyngeal and rectal swabs. N. meningitidis isolates were evaluated for antibiotic susceptibility and characterised by whole genome sequencing. Genetic relationships among the meningococcal carriage isolates were determined using core genome multilocus sequence typing analysis. The soluble domain of AniA (sAniA) protein expression by western blotting was also evaluated.

Results A total of $62(14.1 \%, 95 \% \mathrm{Cl} 11.1$ to 17.6$)$ carriage meningococci were found among $441 \mathrm{MSM}$. Forty-three viable $N$. meningitidis isolates were cultivated (42 from pharyngeal and 1 from rectal swabs). All the viable isolates were susceptible to cefotaxime, ceftriaxone, ciprofloxacin and rifampicin. Four isolates were penicillin G-resistant and $73 \%$ of those penicillin Gsusceptible showed a minimum inhibitory concentration from $0.064 \mu \mathrm{g} / \mathrm{mL}$ to $0.25 \mu \mathrm{g} / \mathrm{mL}$. Serogroup B was the most frequent (44.2\%), followed by $Z(16.3 \%), E(9.3 \%)$, and $Y$ and $W(2.3 \%)$, respectively. Multilocus sequence typing analysis identified 29 sequence types belonging to 12 clonal complexes. The sAniA protein was expressed in 8 out of $28(29 \%)$ screened meningococcal carriage isolates.

Conclusions Serogroup B meningococcal carriage identified from oral and anal specimens among healthy MSM was the most frequent serogroup identified in this study. Molecular evaluation revealed a degree of similarity among strains belonging to the same clonal complex.

\section{INTRODUCTION}

Neisseria meningitidis (Gram-negative diplococcus) is responsible globally for invasive meningococcal disease (IMD), in particular septicaemia and meningitis. Humans are the only hosts of $N$. meningitidis and can carry them harmlessly in the pharynx. ${ }^{1} \mathrm{~N}$. meningitidis can be transmitted through respiratory droplets from patients with IMD or from carriers. ${ }^{1}$
N. meningitidis is transmitted from person to person, and close contact with a healthy carrier can facilitate the spread of the bacteria to susceptible individuals. ${ }^{2}$ Meningococcal carriage was estimated to occur in $10 \%$ of the general population, with most people becoming a carrier multiple times in their lifetime and may carry the bacteria for several months. ${ }^{1}$ Meningococcal carriage increases in teenagers, with peak in young adults at $24 \%-25 \%$. $^{3}$ Carriage status increases in smokers, closed and semiclosed populations, such as military recruits and university students, and in household contacts of a case of meningococcal disease. ${ }^{45}$

Meningococci can cross the epithelial barrier of the pharynx and gain access to the bloodstream, causing septicaemia. Moreover, it can penetrate the blood-brain barrier, giving rise to meningitis. ${ }^{2}$

The human pharynx remains the site preferentially colonised by $N$. meningitidis. Although $N$. meningitidis is an aerobic bacterium, it can respond to oxygen limitation condition by inducing activation of the alternative pathway of denitrification, which is initiated by the copper-containing nitrite reductase (AniA). ${ }^{6}$ Therefore, occasionally urogenital and rectal meningococcal colonisation has been reported, with evidence of transmission between sexual partners involving the pharynx, urogenital tract and rectum. ${ }^{7}$ Molecular characterisation studies have been carried out to understand the epidemiology and the spread of this invasive meningococcal population. ${ }^{89}$ Outbreaks of IMD among men who have sex with men (MSM) have been reported $^{10-13}$; however, few data are available on meningococcal carriage in MSM. ${ }^{1415}$

Based on a screening programme for N. gonorrhoeae conducted during 2016 at the San Gallicano Institute, Rome, Italy, we evaluated the prevalence of $N$. meningitidis carriage isolates among healthy MSM, and the antimicrobial susceptibility and the molecular characteristics, by whole genome sequencing (WGS), and the expression of AniA protein among the analysed isolates.

\section{MATERIALS AND METHODS}

\section{Study population and sample management}

MSM attending, from January to December 2016, the STI/HIV Centre of the San Gallicano Dermatological Institute Hospital, Rome, Italy, were 
screened for N. gonorrhoeae infection by pharyngeal and rectal swabs. N. meningitidis isolates from the same plates used for $N$. gonorrhoeae growth were identified. All meningococcal isolates, with a pseudoanonymous identification (ID) code, were sent to the National Reference Laboratory (NRL) for IMD at the Istituto Superiore di Sanità for further characterisation.

\section{Antimicrobial susceptibility test}

Susceptibility to cefotaxime, ceftriaxone, ciprofloxacin, penicillin $\mathrm{G}$ and rifampicin was determined on all viable meningococcal carriage isolates using the minimum inhibitory concentration (MIC) test strip method (Liofilchem, Italy) on Mueller-Hinton agar (Thermo Scientific, USA), supplemented with 5\% sheep blood. The breakpoints were those recommended by the European Committee on Antimicrobial Susceptibility Testing version 11.0 (1 January 2021; http://eucast.org/).

\section{Beta-lactamase test}

The presence of the bacterial enzyme beta-lactamase was determined on all viable N. meningitidis isolates by rapid acid metric stick method (Oxoid, Italy) on IsoVitaleX 2\% Thayer-Martin agar plate incubated overnight in $5 \%$ carbon dioxide $\left(\mathrm{CO}_{2}\right)$ atmosphere at $37^{\circ} \mathrm{C}$.

\section{Molecular characterisation}

Genomic DNA extraction and WGS

Genomic DNA from cultivated meningococcal isolates $(n=43)$ was extracted using QIAamp DNA Mini Kit (Qiagen, Hilden, Germany) according to the manufacturer's instructions and was sent to the Army Medical Center Scientific Department for WGS. For each isolate, $1 \mathrm{ng}$ of DNA was used to prepare the sequencing libraries, following the Nextera XT DNA protocol, according to the manufacturer's instructions (Illumina, California, USA). WGS was performed on meningococcal carriage isolates using Illumina MiSeq Sequencer Kit (V.3; 600 cycles). On average, 1.9 million paired-end reads were obtained for each sample. A first quality check of the raw sequence data was performed using FastQC. ${ }^{16}$ Reads were trimmed to keep high-quality bases (Q score $>25$ ) using the Sickle V.1.33 software. ${ }^{17}$ Sickle is a slidingwindow, adaptive, quality-based trimming tool for FastQ files (V.1.33 software; available at https://github.com/najoshi/sickle). De novo assembly was carried out with the ABySS V.1.5.2 software $(\mathrm{k}$ parameter $=63) .{ }^{18}$ Contigs longer than $500 \mathrm{bp}$ were selected using an ad hoc script and kept for further analysis. The final assembly ranged from 92 to 847 (median=211) contigs per sample covering $\sim 2.2 \mathrm{Mb}$ of the $N$. meningitidis genome.

\section{Genome analysis}

Of the 43 genomes from carriage isolates, only 32 were uploaded to the PubMLST Neisseria database (http://pubmlst. org/neisseria/). Genogroup definition, PorA and FetA typing, and multilocus sequence typing (MLST) were determined through the meningococcal typing website (http://pubmlst. org/neisseria/). Such information defined the finetype and the genotype as follows: genogroup: PorA(1.)VR1,VR2:FetA(F) VR:ST(cc).

WGS was used to identify penA and $r p o B$ alleles, serogroup $B$ vaccine antigen variants, and aniA allele.

Genomes were compared by means of the Bacterial Isolate Genome Sequence Database (BIGSdb) Genome Comparator Tool $^{19}$ within the PubMLST Neisseria database through geneby-gene analysis. Phylogenetic analysis was performed using the $N$. meningitidis core genome MLST (cgMLST) scheme
(1605 loci) ${ }^{20}$ on 32 carriage isolates of this study, plus 7 invasive meningococci isolated during the study period (2016) in Rome and collected by the NRL within the National Surveillance System for IMD. These invasive meningococci were two serogroup B/cc1572 (ID41657 and ID52942), one serogroup $\mathrm{B} / \mathrm{cc} 32$ (ID41658), one serogroup B/cc213 (ID52946), two serogroup C/cc11 (ID52933 and ID52934), and one serogroup C/cc334 (ID52961).

Incomplete loci were removed automatically from the distance matrix calculation for the neighbour-net graphs. The resulting output distance matrix was used to generate neighbour-net networks using SplitsTree4 software (V.4.13.1).

\section{Western blotting}

To determine the expression of AniA protein, western blotting assay was performed on a subsample of 28 meningococcal carriage isolates, according to previously described method, with minor modifications. ${ }^{21}$ A polyclonal antiserum (Bio-Fab Research, Italy) raised against the soluble domain of AniA protein (sAniA) of $N$. meningitidis serogroup B reference strain MC58 was used for experiments.

Meningococcal isolates were cultured in standard growth conditions at $37^{\circ} \mathrm{C}$ with $5 \% \mathrm{CO}_{2}$ on IsoVitaleX $2 \%$ ThayerMartin plates. Whole cell lysates and supernatants were prepared with 5\% Laemmli buffer. Sodium dodecyl solphatepolyacrilamide gel electrophoresis (SDS-PAGE) was performed on $12 \%$ polyacrylamide gel. Polyclonal rabbit antiserum against AniA protein was used as primary antibody at 1:1000 dilution, and the anti-rabbit IgG conjugated to alkaline phosphatase (Merck Life Science, Italy) was used as secondary antibody at 1:15 000 dilution.

Finally, 5-bromo-4-chloro-3-indolyl-phosphate/nitro blue tetrazolium (BCIP/NBT) Alkaline Phosphatase Substrate (Merck Life Science) was used to visualise the sAniA protein. N. meningitidis MC58 strain was used as the control strain in all experiments.

\section{RESULTS}

\section{Demographic and clinical data}

Sixty-two $(14.1 \%$, 95\% CI 11.1 to 17.6$) N$. meningitidis isolates were identified from 441 MSM. Among them, 43 viable $N$. meningitidis isolates, 42 from pharyngeal and 1 from rectal swabs, were analysed. The median age of the subjects was 36 years (IQR: 23-52 years). All except for two subjects were Italians and confirmed as negative for other STIs.

\section{Antimicrobial susceptibility}

Antimicrobial susceptibility was performed on 37 out of $43 \mathrm{~N}$. meningitidis carriage isolates. All of them were susceptible to cefotaxime, ceftriaxone, ciprofloxacin and rifampicin. Among the isolates susceptible to penicillin G (97\%, 33 of 37), 27 showed MIC for penicillin G higher than $0.064 \mu \mathrm{g} /$ $\mathrm{mL}(0.064 \mu \mathrm{g} / \mathrm{mL}<\mathrm{MIC}<0.25 \mu \mathrm{g} / \mathrm{mL})$ and 4 were penicillin G-resistant $\left(\operatorname{Pen}^{\mathrm{R}}\right)$, with MIC values ranging from $0.38 \mu \mathrm{g} /$ $\mathrm{mL}$ to $0.50 \mu \mathrm{g} / \mathrm{mL}$. All four penicillin-resistant isolates were beta-lactamase-negative.

\section{Molecular characterisation}

The meningococcal genogroup was identified in the 43 meningococcal carriage isolates, with 19 (44\%) serogroup B (MenB), 7 (16\%) MenZ, 4 (9\%) MenE, 1 MenY and 1 MenW. A total of seven isolates (16\%) were $\mathrm{cnl}$ and four (9\%) were nongroupable (NG). 
Table 1 Molecular characteristics of 43 meningococcal carriage isolates

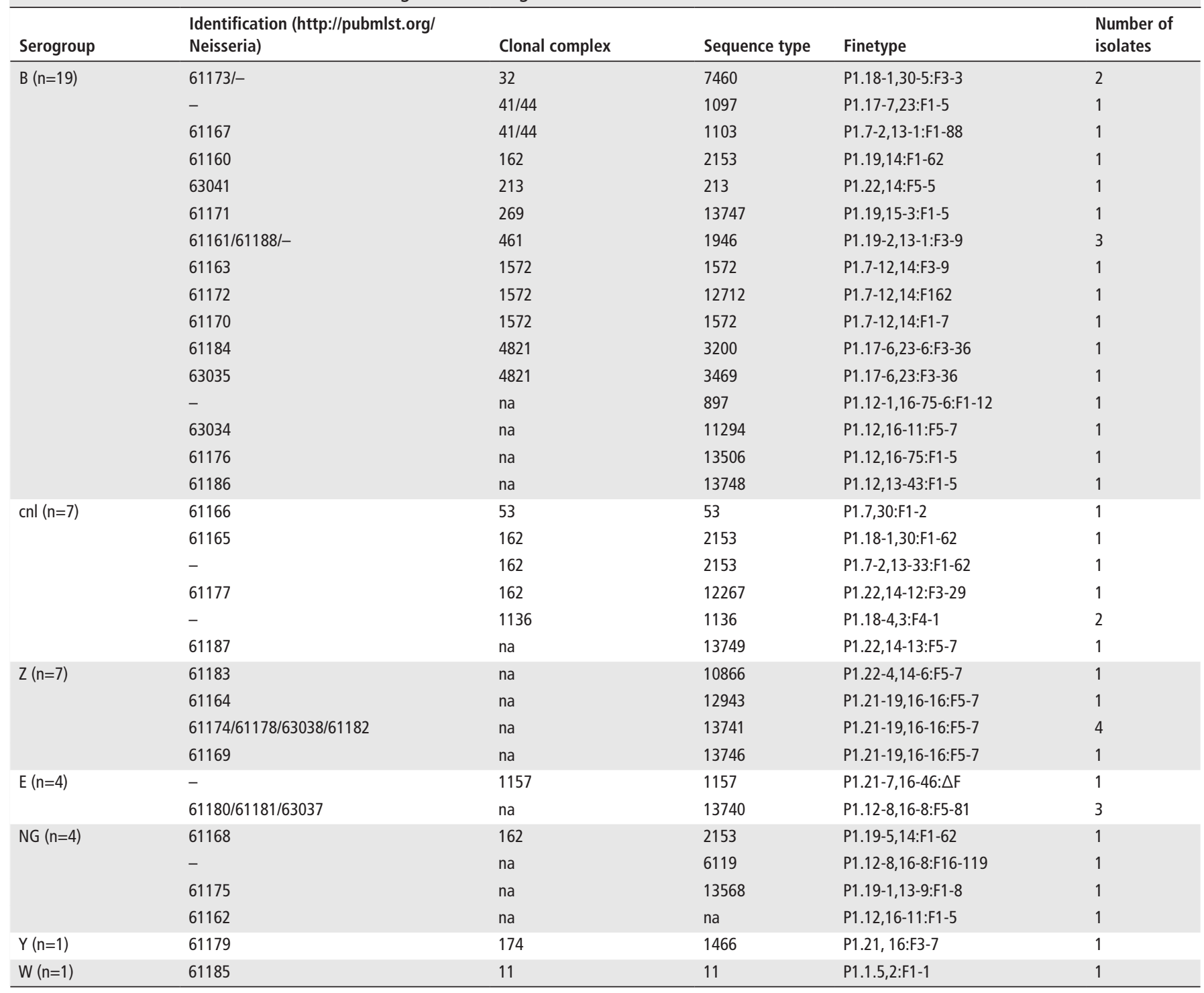

The table was created by the authors.

'-' indicates no genome available.

'na' indicates not assigned to any known clonal complex.

$\mathrm{cnl}$, capsule null locus; $N G$, non-groupable; $\Delta \mathrm{F}$, fetA gene deletion.

MLST identified 29 sequence types (STs) belonging to 12 clonal complexes $\left(\mathrm{cc}_{\mathrm{s}}\right)$, of which cc162 $(\mathrm{n}=5,42 \%)$ and $\mathrm{cc} 461$ $(n=3,25 \%)$ were the most frequent. Twelve sequence types, identified in 12 isolates (4 MenB, 4 MenZ, $2 \mathrm{NG}, 1 \mathrm{MenE}$ and $1 \mathrm{cnl}$ ), were not designed to any known clonal complex and were classified as 'not assigned' (na) (table 1).

Among the MenB isolates, cc461 $(n=3), \operatorname{cc} 1572(n=3)$, $\operatorname{cc} 32(n=2), \operatorname{cc} 41 / \operatorname{cc} 44(n=2), \operatorname{cc} 4821(n=2), \operatorname{cc} 162(n=1)$, $\operatorname{cc} 213(n=1)$ and $\operatorname{cc} 269(n=1)$ were found. Only one of the four MenE isolates belonged to cc1157; the MenW isolate belonged to cc11 and the MenY isolate belonged to cc174. Among the $c n l$ isolates, $c c 162(n=3), \operatorname{cc} 1136(n=2)$ and $\operatorname{cc} 53$ $(n=1)$ were identified (table 1$)$. A total of 29 PorA types and 16 FetA variants were identified. The most frequent PorA types were P1.7-12,14 (3 of 43, 7\%) and P1.21-19,16-16 (3 of $43,7 \%$ ). The predominant FetA variant was F1-62 (6 of 43, $14 \%$ ) and F5-7 (6 of 43, 14\%). One carriage isolate (1 of 43, 2.3\%) gave negative PCR results for fetA gene (table 1).

\section{Meningococcal serogroup $B$ vaccine antigen variants}

4CMenB variant family 2/Pfizer subfamily A (fHbp-2/A) was the most represented (49\%, 21 of 43$)$, followed by variant family $1 /$ Pfizer subfamily B (fHbp-1/B) (21\%, 9 of 43) and variant family 3/subfamily A (fHbp-3/A) (7\%, 3 of 43). Figure 1 shows the fHbp peptides distributed per clonal complex. In all carriage isolates, 19 Neisserial Heparin Binding Antigen (NHBA) peptides were found (figure 2). The most frequent NHBA peptides identified were peptide 20 identified in 7 isolates $(5 \mathrm{cnl} / \mathrm{cc} 162 ; 2 \mathrm{MenB} /$ cc1572); peptide 101 identified in 6 MenZ isolates (4 ST-13741; 1 ST-12943; 1 ST-13746); and NHBA peptide 1172 was detected in 4 isolates (3 MenE ST-13740 and 1 NG ST-6119) (table 1).

The nadA gene was found in eight isolates: NadA-1 in two MenB/cc32 isolates; NadA-8 in three MenZ/ST-13741 isolates, in one MenZ/ST-13746 isolate and in one MenW/ cc11 isolate; and NadA-79 in one $\mathrm{MenB} / \mathrm{cc} 213$ isolate. The remaining isolates (35 of $43,81 \%$ ) resulted negative to the nadA gene. 


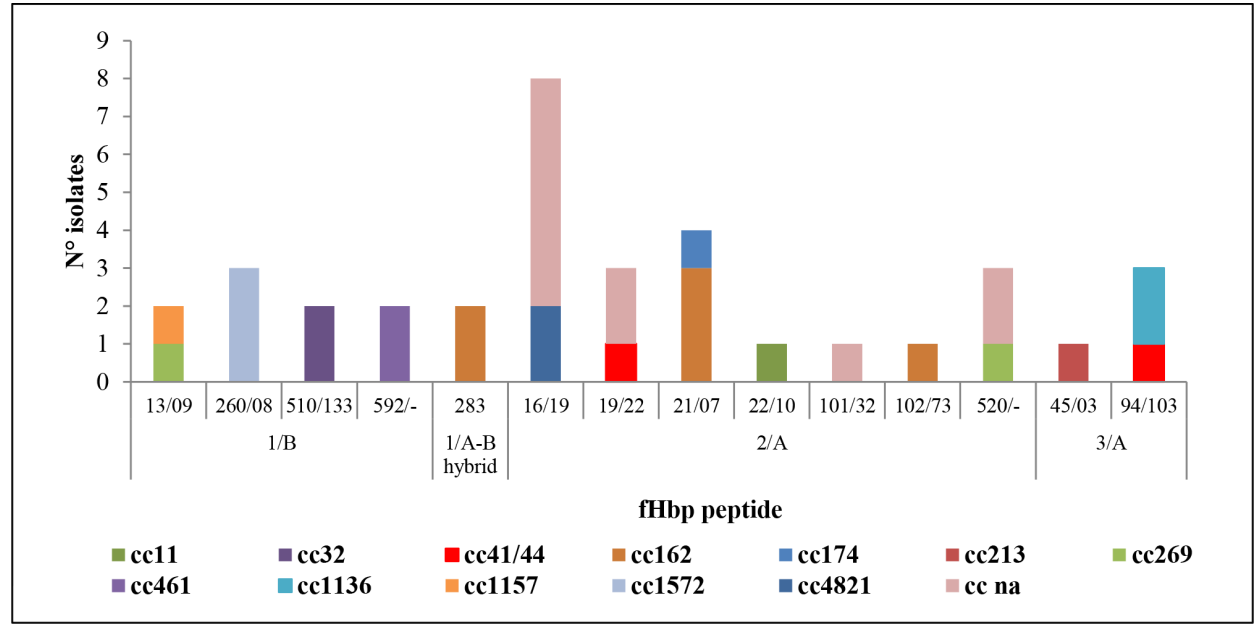

Figure 1 Distribution of fHbp peptides by clonal complex (for each the variant is indicated for each fHbp peptide is indicated its variant). 'na' indicates not assigned to any known clonal complex in the MLST website. cc, clonal complex; MLST, multilocus sequence typing.

Twenty-one PorAVR2 peptides were identified in 34 isolates. The most frequent PorAVR2 peptide was P1.14 $(n=6)$, followed by P1.16-6 ( $\mathrm{n}=3)$. Six PorA VR2 peptides (P1.16-75, P1.23, P1.30, P1.13-1, P1.13-9, P1.16-11) occurred twice and the remaining 15 PorAVR2 peptides occurred once.

\section{pen $A$ and $r p o B$ alleles}

Identification of penA and $r p o B$ alleles was performed on 43 meningococcal carriage isolates. A total of 16 different penA alleles were found. Four penA alleles were identified in the four isolates resistant to penicillin G, of which penA179, penA209 and penA295 were identified in the isolate with MIC of $0.38 \mu \mathrm{g} /$ $\mathrm{mL}$ and penA327 in the isolate with MIC of $0.5 \mu \mathrm{g} / \mathrm{mL}$.

The two prevalent $r p o B$ alleles were $r p o B 21$ (10 of 43, 23\%) and rpoB28 (9 of 43, 21\%), followed by rpoB2 (5 of 43, 12\%), rpoB5 (4 of 43, 9\%), rpoB1 (3 of 43, 7\%), rpoB4 (3 of 43, 7\%), rpoB207 (3 of 43, 7\%), rpoB18 (3 of 43, 7\%) and rpoB85 (2 of 43, 5\%). rpoB8, rроB9, rрoB34 and $r p o B 42$ were detected each one in one isolate.

\section{Core genome multilocus sequence typing}

Thirty-two genomes of 43 meningococcal carriage isolates and 7 invasive meningococcal genomes were analysed using cgMLST to characterise the phylogenetic correlations. The cgMLST analysis included 1269 of the 1605 core genome loci, excluding 336 incomplete loci. All meningococcal carriage and invasive genomes were distributed based on clonal complex or sequence type (figure 3 ). The genome comparison revealed similarities and close relationships among genomes from meningococcal carriage isolates. The two MenZ/ST-13741 (ID63038 and ID61182) had no different loci. Moreover, these two genomes differed by 15 loci with ID61178 (MenZ/ST-13741) and by 55 loci with ID61174 (MenZ/ST-13741). Other two MenZ ID61164 (ST12943) and ID61169 (ST-13746) showed a distance of 17 loci. The three MenE/ST-13740 genomes showed close relationships: between ID63037 and ID61180, isolated from the pharynx and rectal swabs of the same patient, respectively, a distance of 41 different loci were found. Moreover, ID61180 showed only three different loci with ID61181.

The two cc162 genomes from carriage isolates (ID61160 and ID61168) revealed a small genetic distance of 19 loci.

We observed that meningococcal carriage and invasive genomes, belonging to the same clonal complex, grouped together (figure 3). In particular, the two MenB/cc213 genomes (ID63041 from carriage isolate and ID52946 from invasive isolate) clustered together with a distance of 37 loci.

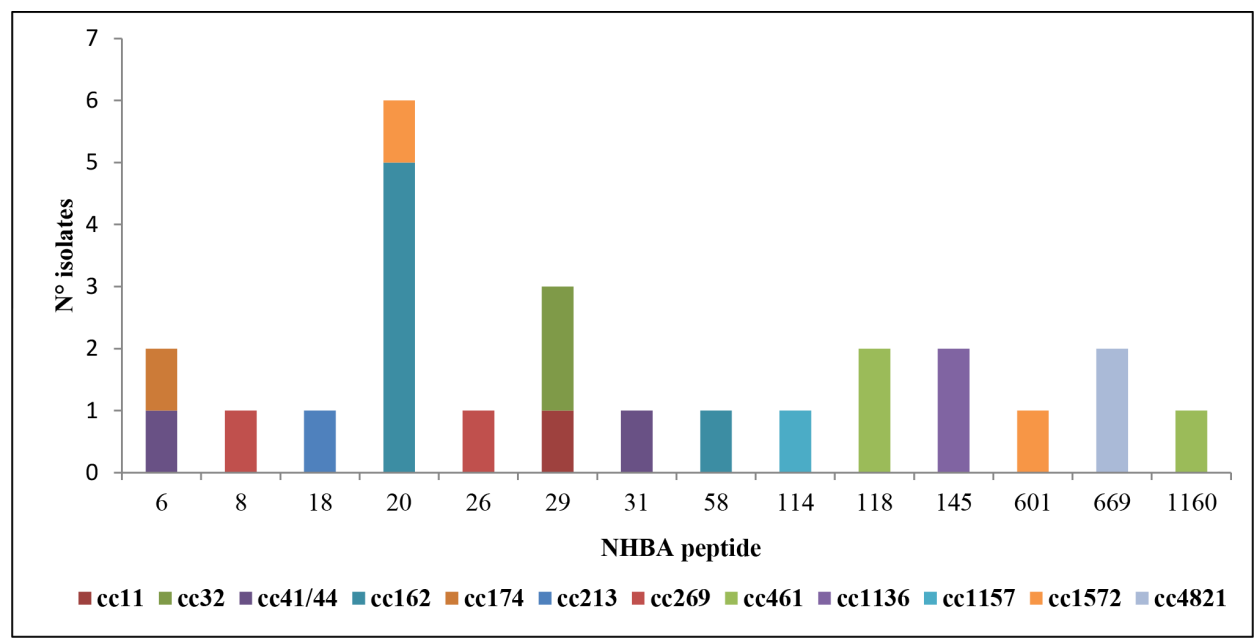

Figure 2 Distribution of NHBA peptides by clonal complex (cc). 


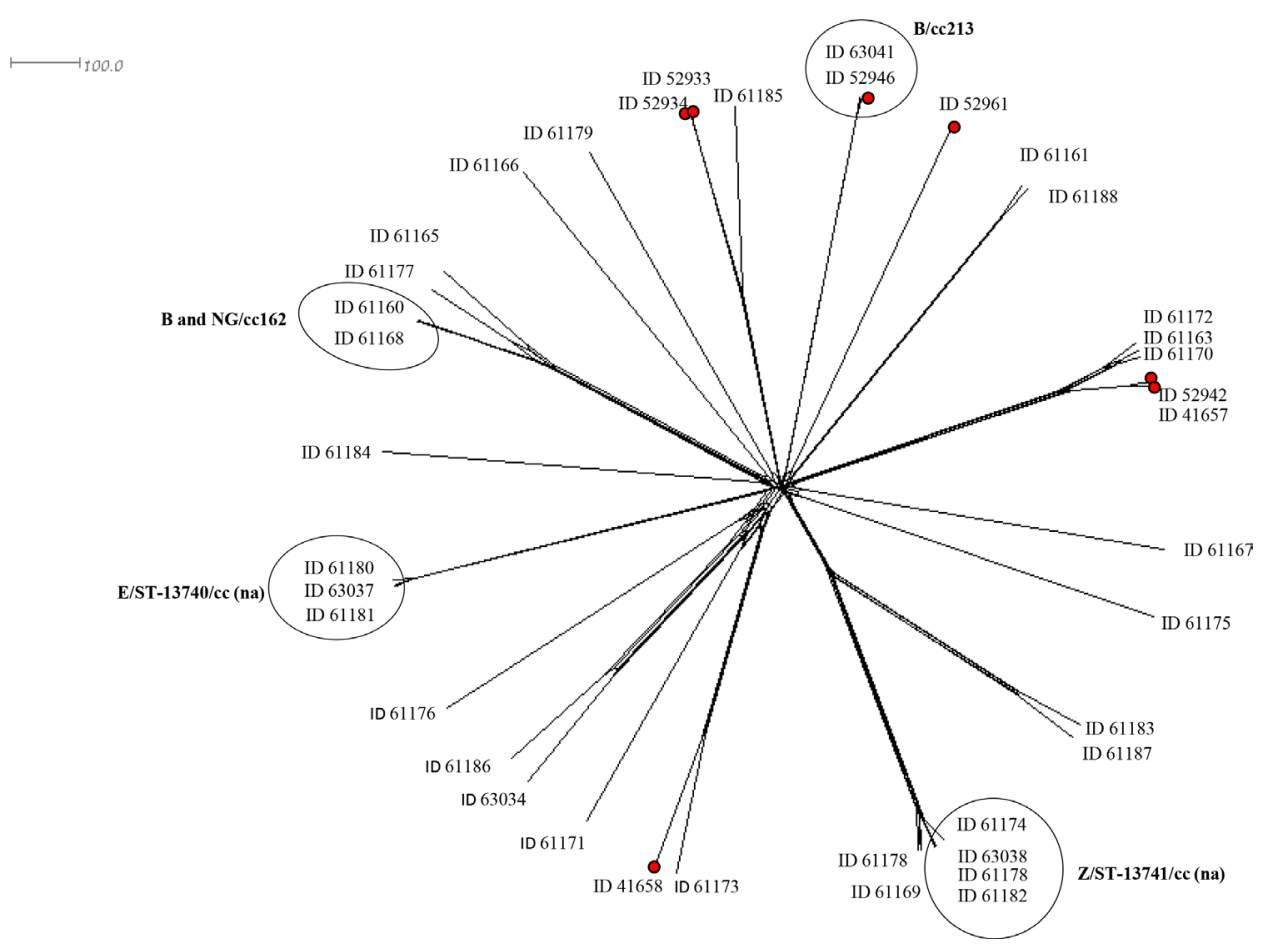

Figure 3 Neighbour-net network based on comparison of Neisseria meningitidis carriage $(n=32)$ and invasive (red dots, $n=7)$ genomes by cgMLST. For each genome the identification (ID) code from http://pubmlst.org/Neisseria is reported. The main clusters among carriage genomes are highlighted by circles. The scale bar represents the number of variant loci. 'na' indicates not assigned to any known clonal complex. cc, clonal complex; cgMLST, core genome multilocus sequence typing.

\section{Western blotting}

As shown in table 2, 8 out of 28 (29\%) screened meningococcal carriage isolates on western blot assay for the expression of sAniA showed the $\sim 55 \mathrm{kDa}$ sAniA form. These isolates were MenE $(n=3)$, MenB $(n=2)$, MenY $(n=1)$, MenZ $(n=1)$ and cnl $(\mathrm{n}=1)$. The molecular characterisation evidenced six aniA alleles: 6, 11, 53, 229, 673 and 675. The aniA allele $675(\mathrm{n}=3)$ was found in three MenE/ST-13740 genomes (na).

AniA protein was not expressed in the remaining 20 isolates (71\%). In four meningococcal isolates, the aniA gene was missing. In one isolate belonging to the genotype W:P1.5,2:F1-1:ST-11 (cc11), a premature stop codon was found in the aniA gene (aniA allele 1) (table 2). The remaining 15 isolates harboured an intact aniA gene but did not express the protein.

\section{DISCUSSION}

Isolation of invasive $N$. meningitidis from MSM has been already reported in several studies, ${ }^{11-13}$ and an increasing number of outbreaks of meningococcal disease has been reported in Europe and in the USA in the same population. ${ }^{13-15} 2223$

In this study, a cohort of MSM participants at a screening programme for $N$. gonorrhoeae infection were considered for the presence of N. meningitidis in the oropharynx and urogenital tracts.

The proportion of meningococcal carriage (14.1\%) was consistent with that already described by Carlin et al. ${ }^{14}$ While invasive meningococcal isolates from MSM found in Europe were predominantly serogroup $\mathrm{C},{ }^{11}$ this serogroup was absent among our carriage isolates. Majority of the carried meningococci were of serogroup $\mathrm{B}$, one of the two most frequent serogroups of invasive meningococcal isolates circulating in Italy in the same period (https://www.iss.it/sn-mbi-rapporti-iss).
The clonal complexes of the carriage meningococci analysed accounted for the most frequently clonal complexes reported in healthy carriers. ${ }^{34}$ Only three carriage isolates belonged to the hypervirulent clonal complexes (cc41, cc44 and cc11), mainly associated with invasive meningococci circulating in the country. ${ }^{24}$

Phylogenetic analysis to investigate the genetic relationships among these carriages and invasive meningococci, isolated in Rome in the same period, revealed clusters including isolates belonging to the same clonal complexes regardless of the invasiveness. We can hypothesise that carriage meningococci belonging to a hypervirulent clonal complex may be widely transmitted in the MSM community and may determine an invasive disease. Moreover, the high degree of genetic similarity for carriage isolates belonging to the same clonal complex may suggest the presence of a cluster of those isolates among the screened MSM.

Evaluation of antibiotic susceptibility revealed a high proportion of carriage isolates with reduced susceptibility to penicillin $G$ and resistance to penicillin $G$ in four carriage isolates. Among the resistant carriage isolates to penicillin $G$, one penA327 allele was already described in the meningococcal isolates from the outbreak of IMD among MSM..$^{25}$ This allele is identical to the allele penAXXXIV, associated with resistance to third-generation cephalosporin and reduced susceptibility to cefotaxime in N. gonorrhoeae. ${ }^{25}$ However, penA327 allele was associated with resistance to penicillin $G$, but not with resistance to cephalosporin and reduced susceptibility to cefotaxime. These results correlate with the possibility of a genetic exchange among gonococci and carriage meningococci in the pharynx, as already described in MSM. ${ }^{26}$ Because of this evidence, all the individuals identified as meningococcal carriers were recalled at the San Gallicano Dermatological Institute and subjected to 
Table 2 Genogroup, genotype, aniA allele and AniA expression detected in 28 meningococcal carriage isolates from men who have sex with men

\begin{tabular}{|c|c|c|c|c|}
\hline Genogroup & $\begin{array}{l}\text { Identification (http://pubmlst.org/ } \\
\text { Neisseria) }\end{array}$ & Genotype & aniA allele & AniA protein \\
\hline \multirow[t]{12}{*}{$B(n=12)$} & 61173 & B:P1.18-1,30-5:F3-3:ST-7460 (cc32) & 229 & + \\
\hline & 61167 & B:P1.7-2,13-1:F1-88:ST-1103 (41/44) & 2 & - \\
\hline & 63041 & B:P1.22,14:F5-5:ST-213 (cc213) & 19 & - \\
\hline & 61171 & B:P1.19,15-3:F1-5:ST-13747 (cc269) & 4 & - \\
\hline & 61161 & B:P1.19-2,13-1:F3-9:ST-1946 (cc461) & 673 & + \\
\hline & 61163 & B:P1.7-12,14:F3-9:ST-1572 (cc1572) & $x$ & - \\
\hline & 61170 & B:P1.7-12,14:F3-9:ST-1572 (cc1572) & $x$ & - \\
\hline & 61172 & B:P1.7-12,14:F162:ST-12712 (cc1572) & $x$ & - \\
\hline & 61184 & B:P1.17-6,23-6:F3-36:ST-3200 (cc4821) & 228 & - \\
\hline & 63035 & B:P1.17-6,23:F3-36:ST-3469 (cc4821) & 8 & - \\
\hline & 63034 & B:P1.12,16-11:F5-7:ST-11294 (UNK) & 4 & - \\
\hline & 61176 & B:P1.12,16-75:F1-5:ST-13506 (UNK) & 674 & - \\
\hline \multirow[t]{3}{*}{$\mathrm{cnl}(\mathrm{n}=3)$} & 61166 & cnl:P1.7,30:F1-2:ST-53 (cc53) & 17 & - \\
\hline & 61165 & cnl:P1.18-1,30:F1-62:ST-2153 (cc162) & 6 & + \\
\hline & 61187 & cnl:P1.22,14-13:F5-7:ST-13749 (UNK) & 11 & - \\
\hline \multirow[t]{6}{*}{$Z(n=6)$} & 61183 & Z:P1.22-4,14-6:F5-7-119:ST-10866 (UNK) & 11 & + \\
\hline & 61164 & Z:P1.21-19,16-16:F5-7:ST-12943 (UNK) & 240 & - \\
\hline & 61174 & Z:P1.21-19,16-16:F5-7:ST-13741 (UNK) & 240 & - \\
\hline & 63038 & Z:P1.21-19,16-16:F5-7:ST-13741 (UNK) & 240 & - \\
\hline & 61182 & Z:P1.21-19, 16-16:F5-7:ST-13741 (UNK) & 240 & - \\
\hline & 61169 & Z:P1.21-19,16-16:F5-7:ST-13746 (UNK) & 240 & - \\
\hline \multirow[t]{3}{*}{$E(n=3)$} & 61180 & E:P1.12-8,16-8:F5-81:ST-13740 (UNK) & 675 & + \\
\hline & 61181 & E:P1.12-8,16-8:F5-81:ST-13740 (UNK) & 675 & + \\
\hline & 63037 & E:P1.12-8,16-8:F5-81:ST-13740 (UNK) & 675 & + \\
\hline \multirow[t]{2}{*}{$N G(n=2)$} & 61168 & NG:P1.19-5,14:F1-62:ST-2153 (сc162) & 6 & - \\
\hline & 61175 & NG:P1.19-1,13-9:F1-8:ST-13568 (UNK) & $x$ & - \\
\hline$Y(n=1)$ & 61179 & Y:P1.21,16:F3-7:ST-1466 (сc174) & 53 & + \\
\hline$W(n=1)$ & 61185 & W:P1.1.5,2:F1-1:ST-11 (cc11) & 1 & - \\
\hline
\end{tabular}

The table was created by the authors.

aniA with premature stop codon.

$\mathrm{cnl}$, capsule null locus; NG, non-groupable; UNK, unknown; $\mathrm{X}$, missing aniA gene.

chemoprophylaxis with ceftriaxone $1 \mathrm{~g}$ as a single intramuscular dose. Ceftriaxone was used based on the antibiotic susceptibility of the isolates and in agreement with the guidelines for chemoprophylaxis in contacts of patients with invasive disease. In addition, many of the meningococcal carriers had been already treated with ceftriaxone for previous $N$. gonorrhoeae infection without adverse events. Ceftriaxone can be used at any age and was thus advantageous given the wide age range of the subjects to be treated. Moreover, to reduce meningococcal carriage and the risk of invasive disease, every effort was made to enhance vaccination coverage for $N$. meningitidis among MSM. ${ }^{27}$

The copper-containing nitrate reductase, AniA, plays a significant role in catalysing the conversion of nitrite to nitric oxide, which is subsequently reduced to nitrous oxide by NorB. ${ }^{28}$ The ability of $N$. meningitidis to grow under oxygen limitation through denitrification pathway has facilitated its adaptation to environments where oxygen availability is variable. ${ }^{21}$ While this pathway is crucial to the survival of N. gonorrhoeae, the denitrification pathway is not essential in the survival of $N$. meningitidis in the nasopharynx. ${ }^{29}$ Hereby, $29 \%$ of the carriage meningococci expressed the AniA protein except for 5 isolates, lacking the gene or with a premature stop codon in the first half of the nucleotide sequence, while the other 15 meningococcal isolates were unable to express the protein despite the presence of the entire aniA gene. Since the expression of meningococcal AniA protein is under the control of a complex regulatory network involving multiple transcriptional regulators, it is possible to hypothesise that nucleotide polymorphisms in the putative regulator sites explain the inability of these meningococci to produce AniA. ${ }^{29}$

A report published by Taha et $a l^{11}$ revealed that meningococcal isolates collected from the urethra harboured a functional AniA, but were defective for $\mathrm{fHbp}$ due to a premature stop codon. This phenotype $\left(\mathrm{AniA}^{+}, \mathrm{fHbp}^{-}\right)$could reflect an adaptation and selection of meningococci which colonised uncommon sites, such as the urethra and the rectum. ${ }^{11}$ This result differs from the data here presented, with majority of the isolates from the pharynx harbouring an entire $\mathrm{fH} b \mathrm{p}$ gene.

\section{CONCLUSION}

Our findings, together with data collected from the literature, underline the appropriateness of meningococcal screening in the MSM community, to adopt the opportune clinical practices and public health action.

Moreover, our study highlights the need to further characterise meningococci isolated from the pharyngeal and anorectal tracts, particularly in terms of the new genes they can acquire, including those classified as antibiotic resistance determinants. It is important to identify the meningococcal serogroup to assess how and if these strains may be preventable by vaccination. Information on serogroups could be essential in the implementation of public health control measures in case of an outbreak, 
including vaccination, which has been reported to manage an outbreak of IMD of serogroup C in MSM in New York City. ${ }^{23}$

\section{Key messages}

- Serogroup B was the main serogroup identified among the meningococci isolated from healthy men who have sex with men (MSM) in this study.

- The acquisition of penA327 allele in one penicillin G-resistant meningococcal carriage isolate highlights the possibility of exchange, in the pharynx, of genetic materials among meningococci and gonococci.

- This study underlines the importance of pharyngeal and rectal swab screening to detect Neisseria meningitidis among MSM.

\section{Handling editor Federico Garcia}

Twitter Massimo Giuliani @giumas3

Acknowledgements This publication made use of the PubMLST website (https:// pubmlst.org/) and sited at the University of Oxford. The development of this website was funded by the Wellcome Trust. We thank Keith Jolley from the University of Oxford for editing the text.

Contributors PS, GP and MG designed the study. AN and AP wrote the first draft of the manuscript. AN was the author responsible for the overall content as the guarantor. AP, AN, CF, PV and LA performed the molecular analysis. AC, SF $\mathrm{AA}, \mathrm{AF}$ and $\mathrm{RL}$ carried out the whole genome sequencing. All authors reviewed and commented critically to the manuscript and gave final approval to submit for publication.

Funding The authors have not declared a specific grant for this research from any funding agency in the public, commercial or not-for-profit sectors.

Competing interests None declared.

Patient consent for publication Consent obtained directly from patient(s).

Ethics approval The study was cleared by the Institutional Ethics Committee, San Gallicano Dermatological Institute, IRCSS IFO Section-Fondazione GB Bietti (CE/626/11, 28-11-2011).

Provenance and peer review Not commissioned; externally peer reviewed.

Data availability statement All data relevant to the study are included in the article or uploaded as supplementary information.

Open access This is an open access article distributed in accordance with the Creative Commons Attribution Non Commercial (CC BY-NC 4.0) license, which permits others to distribute, remix, adapt, build upon this work non-commercially, and license their derivative works on different terms, provided the original work is properly cited, appropriate credit is given, any changes made indicated, and the use is non-commercial. See: http://creativecommons.org/licenses/by-nc/4.0/.

ORCID iD

Arianna Neri http://orcid.org/0000-0001-5748-880X

\section{REFERENCES}

1 Hill DJ, Griffiths NJ, Borodina E, et al. Cellular and molecular biology of Neisseria meningitidis colonization and invasive disease. Clin Sci 2010;118:547-64.

2 Caugant DA. Genetics and evolution of Neisseria meningitidis: importance for the epidemiology of meningococcal disease. Infect Genet Evol 2008;8:558-65.

3 Neri A, Fazio C, Ambrosio L, et al. Carriage meningococcal isolates with capsule null locus dominate among high school students in a non-endemic period, Italy, 20122013. Int J Med Microbiol 2019;309:182-8

4 Bogaert D, Hermans PWM, Boelens $\mathrm{H}$, et al. Epidemiology of nasopharyngeal carriage of Neisseria meningitidis in healthy Dutch children. Clin Infect Dis 2005;40:899-902.
5 Tryfinopoulou K, Kesanopoulos K, Xirogianni A, et al. Meningococcal carriage in military recruits and university students during the pre MenB vaccination era in Greece (2014-2015). PLoS One 2016;11:e0167404.

6 Anjum MF, Stevanin TM, Read RC, et al. Nitric oxide metabolism in Neisseria meningitidis. J Bacteriol 2002;184:2987-93.

7 Ladhani SN, Lucidarme J, Parikh SR, et al. Meningococcal disease and sexual transmission: urogenital and anorectal infections and invasive disease due to Neisseria meningitidis. Lancet 2020;395:1865-77.

8 Odegaard K, Gundersen T. Isolation of Neisseria meningitidis in urogenital/rectal infections. Acta Derm Venereol 1977:57:173-6.

9 Chapel TA, Gatewood C, Keane MB. Neisseria meningitidis in the anal canal of homosexual men.J Infect Dis 1977:136:810-2.

10 Kanemitsu N, Hayashi I, Satoh N, et al. Acute urethritis caused by Neisseria meningitidis. Int J Urol 2003:10:346-7.

11 Taha M-K, Claus H, Lappann M, et al. Evolutionary events associated with an outbreak of meningococcal disease in men who have sex with men. PLoS One 2016;11:e0154047

12 Marcus U, Vogel U, Schubert A, et al. A cluster of invasive meningococcal disease in young men who have sex with men in Berlin, October 2012 to may 2013. Euro Surveill 2013;18. doi:10.2807/1560-7917.ES2013.18.28.20523. [Epub ahead of print: 11 Jul 2013].

13 Weiss D, Varma JK. Control of recent community-based outbreaks of invasive meningococcal disease in men who have sex with men in Europe and the United States. Euro Surveill 2013:18:es2013.18.28.20522.

14 Carlin EM, Hannan M, Walsh J, et al. Nasopharyngeal flora in HIV seropositive men who have sex with men. Genitourin Med 1997:73:477-80.

15 Tinggaard M, Slotved H-C, Fuursted K, et al. Oral and anal carriage of Neisseria meningitidis among sexually active HIV-infected men who have sex with men in Denmark 2014-15. Int J Infect Dis 2021;105:337-44.

16 Andrews S. Fast Qc a quality control tool for high throughput sequence data. Cambridge: Babraham Institute, 2010. http://www.bioinformatics.babraham.ac.uk/ projects/fastqc

17 Fass JN, Joshi NA, Couvillion MT, et al. Genome-Scale analysis of programmed DNA elimination sites in Tetrahymena thermophila. G3 2011;1:515-22.

18 Simpson JT, Wong K, Jackman SD, et al. ABySS: a parallel assembler for short read sequence data. Genome Res 2009;19:1117-23.

19 Jolley KA, Maiden MCJ. BIGSdb: scalable analysis of bacterial genome variation at the population level. BMC Bioinformatics 2010;11:595

$20 \mathrm{KC} \mathrm{M}$, Unemo M, Jeverica S. Genomic characterization of Urethritis-Associated Neisseria meningitidis shows that a wide range of $\mathrm{N}$. meningitidis strains can cause urethritis. J ClinMicrobiol 2017:55:3374-83.

21 Stefanelli P, Colotti G, Neri A, et al. Molecular characterization of nitrite reductase gene (aniA) and gene product in Neisseria meningitidis isolates: is aniA essential for meningococcal survival? IUBMB Life 2008;60:629-36.

22 Agrawal A, Jasdanwala S, Agarwal A, et al. Fatal Waterhouse-Friderichsen syndrome due to serotype C Neisseria meningitidis in a young HIV negative MSM (men who have sex with men). BMJ Case Rep 2014:2014:bcr2014206295.

23 Kratz MM, Weiss D, Ridpath A, et al. Community-Based outbreak of Neisseria meningitidis serogroup $C$ infection in men who have sex with men, New York City, New York, USA, 2010-2013. Emerg Infect Dis 2015;21:1379-86.

24 Ambrosio L, Neri A, Fazio C, et al. Genomic analysis of Neisseria meningitidis carriage isolates during an outbreak of serogroup C clonal complex 11, Tuscany, Italy. PLoS One 2019:14:e0217500.

25 Deghmane A-E, Hong E, Taha M-K. Emergence of meningococci with reduced susceptibility to third-generation cephalosporins. J Antimicrob Chemother 2017;72:95-8

26 Zapun A, Morlot C, Taha M-K. Resistance to $\beta$-lactams in Neisseria ssp due to chromosomally encoded penicillin-binding proteins. Antibiotics 2016;5:35.

27 Zalmanovici Trestioreanu A, Fraser A, Gafter-Gvili A, et al. Antibiotics for preventing meningococcal infections. Cochrane Database Syst Rev 2013;2013:CD004785.

28 Barth KR, Isabella VM, Clark VL. Biochemical and genomic analysis of the denitrification pathway within the genus Neisseria. Microbiology 2009:155:4093-103.

29 Ku SC, Schulz BL, Power PM, et al. The pilin 0-glycosylation pathway of pathogenic Neisseria is a general system that glycosylates AniA, an outer membrane nitrite reductase. Biochem Biophys Res Commun 2009;378:84-9. 